\title{
High Speed Shadowgraphy of Transparent Nozzles as an Evaluation Tool for In-Nozzle Cavitation Behavior of GDI Injectors
}

\author{
Dmitrii Mamaikin ${ }^{\star 1}$, Tobias Knorsch¹, Philipp Rogler ${ }^{1}$, Philippe Leick¹, Michael Wensing² \\ ${ }^{1}$ Robert Bosch GmbH, Germany \\ ${ }^{2}$ Department of Engineering Thermodynamics, FAU Erlangen-Nuremberg, Germany \\ ${ }^{*}$ Corresponding author: Dmitrii.Mamaikin@de.bosch.com
}

\begin{abstract}
Gasoline Direct Injection (GDI) systems have become a rapidly developing technology taking up a considerable and rapidly growing share in the Gasoline Engine market due to the thermodynamic advantages of direct injection. The process of spray formation and propagation from a fuel injector is very crucial in optimizing the air-fuel mixture of DI engines. Previous studies have shown that the presence of some cavitation in high-pressure fuel nozzles can lead to better atomization of the fluid. However, under some very specific circumstances, high levels of cavitation can also delay the atomization process; spray stabilization due to hydraulic flip is the most well-known example. Therefore, a better understanding of cavitation behavior is of vital importance for further optimization of next generation fuel injectors.

In contrast to the abundance of investigations conducted on the inner flow and cavitation patterns of diesel injectors, corresponding in-depth research on the inner flow of gasoline direct-injection nozzles is still relatively scarce. In this study, the results of an experiment performed on real-size GDI injector nozzles made of acrylic glass are presented. The inner flow of the nozzle is visualized using a high-power pulsed laser, a long-distance microscope and a highspeed camera. The ambiguity of dark areas on the images, which may represent cavitation regions as well as ambient air drawn into the nozzle holes, is resolved by injecting the fuel both into a fuel or gas filled environment. In addition, the influence of backpressure on the transient flow characteristics of the internal flow is investigated. In good agreement with observations made in previous studies, higher backpressure levels decrease the amount of cavitation inside the nozzles. Due to the high temporal and spatial resolution of the experiment, the transient cavitation behavior during the opening, quasi-steady and closing phases of the injector needle motion can be analyzed. For example, it is found that cavitation patterns oscillate with a characteristic frequency that depends on the backpressure. The link between cavitation and air drawn into the nozzle at the beginning of injection is also revealed.
\end{abstract}

\section{Keywords}

Direct injection, transparent nozzle, internal flow, cavitation, high-speed imaging

\section{Introduction}

The performance of GDI engines depends significantly on the fuel injection system. The injector plays a dominant role in delivery of the fuel into the combustion chamber in the form of spray patterns. In past years, many studies have been dedicated to the processes of spray formation with the main goal of achieving better control of spray propagation and improved atomization characteristics [1-3]. These factors consequently influence the processes of fuel evaporation, ignition and combustion to a great extent.

The technology development trend towards higher injection pressures makes the investigations of two-phase internal flows highly important, in part due to the possibility of more intense pressure fluctuations inside the nozzle. In addition, it is known that the velocity profile at the nozzle outlet, as well as turbulence intensity and liquid-cavitation interaction inside the nozzle provoke the first perturbations of the liquid stream $[4,5]$. These perturbations directly lead to droplet formation via primary break-up processes but also contribute indirectly to secondary atomization due to increased aerodynamic resistance.

The high speed cavitating flow that occurs inside real-size injector nozzles is quite challenging for experimental studies due to the small relevant scales and the complexity of the highly transient and turbulent flow. Therefore, the cavitating flow is still not fully understood, which in turn obstructs the understanding of some crucial processes such as primary break-up and hole-to-hole spray variations.

Most of the experimental work conducted on internal nozzle phenomena has relied on shadowgraphy. For instance, some studies were carried out on enlarged transparent nozzles [6-8]. The large scale significantly improves the optical accessibility to the flow inside the nozzle. For these measurements, however, the relevant dimensionless parameters such as Reynolds and cavitation numbers should ideally be identical to those of the real-size nozzles 
in order to maintain equivalent flow conditions. Moreover, the Ohnesorge number should be preserved in order to obtain similar break-up of the liquid leaving the nozzle. In most of the cases, it is not possible to meet all these conditions simultaneously and therefore, large scale experiments cannot be fully representative. Other experimental studies have used two-dimensional (2D) nozzles for internal flow analysis [5,9]. Optical measurements are simplified due to minimization of the optical aberrations by virtue of the 2D nozzle geometry. This approach facilitates deep and profound studies of cavitation but the results are not fully transferable to real three dimensional nozzles. Another method for internal flow investigation uses real-size transparent nozzles. This avoids scaling effects and thus comes closer to the inner flow of commercial injector nozzles. However, manufacturing such nozzles out of transparent materials is challenging, since in addition to the small size of the nozzle, some resistance to high-pressure conditions is needed. A number of experimental studies were conducted using single hole axial nozzles [10-12] which simplifies both the manufacturing of the nozzle and the resulting flow. However, it is known that sharp turn the liquid needs to take to enter the spray hole is an important driving factor for the subsequent spray breakup. Additionally, in multi-hole injectors, the individual holes can 'communicate'. Only a few experimental studies have compared flows inside single hole and two-hole nozzles [10,13]. Different string cavitation behavior has been found and cavitation strings from one hole can extend to the neighboring hole. The string cavitation, in turn, highly influences the internal flow dynamics as well as the atomization properties [14,15].

Overall, the simplifications that are often made for internal flow measurements, do not provide fully representative results that can be used for further development of injector nozzles. The relevant boundary conditions, needle movement, time scales of measurements, geometries are not or only partially considered. Therefore, experiments, which are performed as close as possible to real conditions, are of significant interest to the research and engineering communities. In this work, the measurement results from high-speed imaging of the internal flow of two-hole real-size transparent nozzles are presented.

\section{Material and methods}

The experimental setup used for this study is schematically depicted in Figure1. The overall setup consists of the injection and the imaging system. The fuel is stored in a vessel which is connected to a high pressure nitrogen supply on one side and the fuel line feeding the injector from the other side. A Bosch HDEV5 gasoline injector is used for injecting the fuel. Such an injector facilitates the mounting of the acrylic parts and the adjustment of maximum needle lift using carefully selected spacer rings. An in-line filter is mounted right upstream of the injector in order to remove contaminants from the fuel, which could affect cavitation inception, deteriorate image quality or even damage the acrylic components. The injection system works without a fuel pump whose operation might provoke the release of dissolved gases upstream the injector and thereby influence the internal flow. The visualization system is composed of a light source, an optical diffusor and a high-speed camera equipped with a long-distance microscope (LDM). The Cavilux HF laser of $810 \mathrm{~nm}$ wavelength is used to illuminate the flow field. Ultra-short pulses of only 50 ns duration are used instead of continuous illumination in order to avoid motion blur in the images. Additionally, the low-coherence of the light provided by the laser avoids formation of any speckle patterns. The laser light is guided by an optical waveguide towards the acrylic nozzle, but first passes through an optical diffusor in order to illuminate the nozzle homogeneously. A high-speed camera (FASTCAM SA-Z) equipped with a long-distance microscope (Infinity K2 with CF4 lens) is used for the image acquisition and operated at $120,000 \mathrm{fps}$. In this way, a magnification of 7.4 is achieved at an image resolution of $640 \times 216$ pixels. A trigger is used to synchronize the injector, camera gate pulses and the laser illumination.

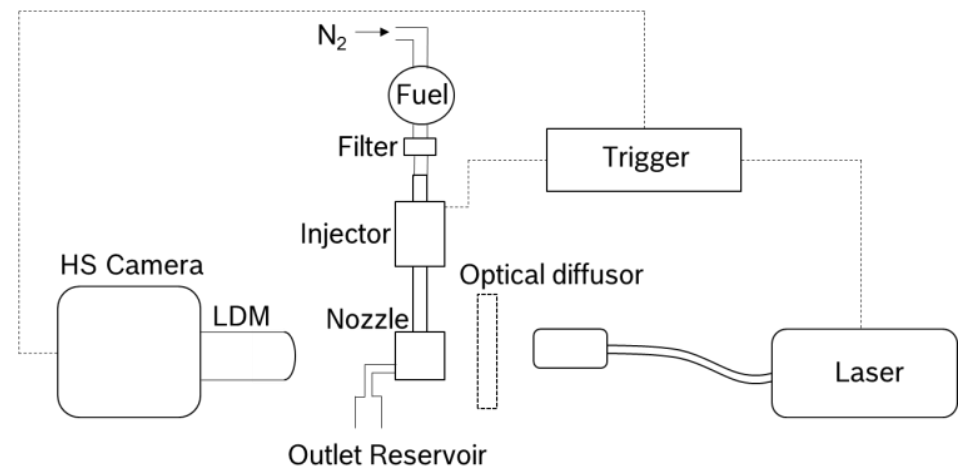

Figure 1. Scheme of the experimental setup. 
The optically accessible nozzle is made of acrylic glass. Figure $2 a$ illustrates the nozzle, where the injector needle and O-ring can also be seen. The spray holes and counterbores are followed by a "multi-step" configuration that allows the fuel to leave the acrylic block and is relatively straightforward to manufacture. The nozzle was tested at maximum injection pressure of 200 bar and showed reliable performance under high pressure load conditions. Due to the configuration of the nozzle, the visualization of the internal nozzle flow is focused on the spray holes area. The spray hole and the counterbore are followed by a "multi-step" outlet that facilitates the withdrawal of fuel from the nozzle exit region before the start of the next injection but does not affect flow and cavitation within the spray hole. The region of interest is outlined with a rectangle in Figure 2a. A typical shadowgraph image captured during a quasi-steady-state of the injection phase is shown in Figure $2 \mathrm{~b}$. As can be seen, the nozzle contains two holes with different inclination angles (I-angles). The I-angle is given with respect to the vertical nozzle axis. A mixture of calibration fluid (ISO 4113 norm) and Bromonaphthalene is used for investigating internal phenomena. This mixture has the same refractive index $(n=1.49)$ as the acrylic glass, which allows an undisturbed view through curved structures. The dark round area on the top of the image is the injector needle, which is opaque to the incident light. The dark areas inside the nozzle holes are gas-phase structures and can either contain fuel vapor (cavitation) or ambient air drawn into the nozzle holes. The refractive index of either of these gases $(n \approx 1)$ is significantly different from the refractive indices of the liquid fuel and the transparent nozzle. Therefore, the incident light is strongly refracted making the corresponding areas to appear as dark regions.

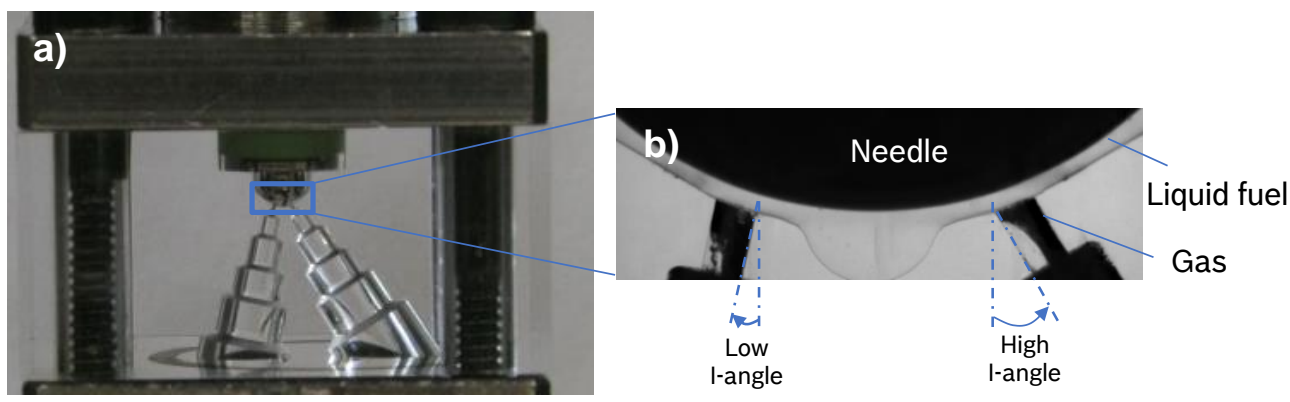

Figure 2. a) Acrylic nozzle configuration, b) Typical shadowgraph image of in-nozzle flow.

In order to resolve the ambiguity of dark areas inside the nozzle holes on shadowgraph images, visualizations are performed while injecting into the fuel into reservoirs filled with air or fuel; in the latter case, no ambient air can be drawn into the system. However, the release of gases dissolved in the fuel still can take place during the fuel injection. It should be pointed out that cavitation and released gases cannot be distinguished in the images. Keeping in mind both possibilities, dark areas will be referred to as 'cavitation' in this paper for the sake of simplicity and easier reading. Figure 3 shows the acrylic nozzle in both air-filled and liquid-filled environments.
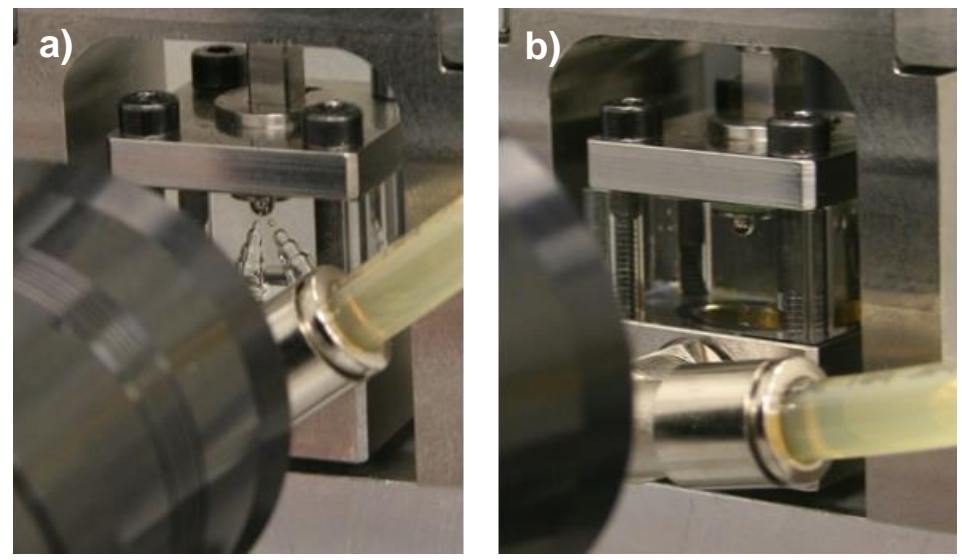

Figure 3. Acrylic nozzle for: a) air-filled nozzle holes, b) fuel-filled nozzle holes

The measurement results of two different acrylic nozzles are presented and discussed in the following sections. One nozzle has two cylindrical holes, whereas another one has two divergent holes. The corresponding holes have identical inclination angles (low I-angle, high I-angle in Figure $2 \mathrm{~b}$ ) but different length to diameter ratios. All the measurements are carried out at room temperature. 


\section{Results and discussion}

Figure 4 shows typical images taken during the measurements while injecting into an air-filled environment. A nozzle with two cylindrical holes was used for this case. The operating conditions for these measurements were as follows: injection pressure - 100 bar, back pressure - 1bar. On the first image (Figure 4a), the needle is closed and one can see stagnant residual air bubbles inside the nozzle holes as well as inside the sac volume. The succeeding image Figure $4 \mathrm{~b}$ is taken during the needle opening phase. The needle lift at this time is still low and as a result, highly turbulent flow occurs inside the nozzle. The holes are filled with gas and one can observe gas pockets, which extend from the nozzle holes and reach the needle. This phenomenon was also observed by Gilles-Birth et al. [10]. It is commonly attributed to strong vorticities in the flow field, which result in a radial pressure drop from the wall to the middle of the hole. In addition, the residual air inside the sac volume is being forced out by the build-up of the liquid flow as can be observed in Figure 4b. The next two images (Figure 4c,d) reveal that the developed cavitation structures inside the nozzle holes maintain their shapes during the steady maximum lift needle position. As it can be seen, cavitation is not formed in the needle seat area. Rather, the cavitation starts at the sharp inlet edges of the nozzle holes. According to the terminology used in [16], 'partial supercavitation' is observed during this stage. It is obvious that the inclination of the nozzle holes affects the location and structure of the in-nozzle cavitation during the steady-state phase of the injection (Figure 4c,d). From the flow point of view, the liquid flow undergoes deflection while it enters the nozzle holes. The flow deflection angle determines the onset of cavitation. In fact, a greater flow deflection angle leads to a higher pressure drop. This, in turn, leads to the cavitation and release of air induced by the nozzle geometry. Therefore, the cavitation is dominant on the right side of both nozzle holes. Figure $4 \mathrm{e}$ represents the nozzle flow during the closing phase of the needle. The amount of cavitation is increased in comparison to the quasi steady state of injection (Figure $4 \mathrm{c}, \mathrm{d}$ ) due to the increased nozzle flow turbulence caused by the needle motion. After the needle has completely closed (Figure 4f), significant amount of gas remains inside the nozzle holes and some gas amount is drawn into the sac volume, which is observed in the form of large bubbles.

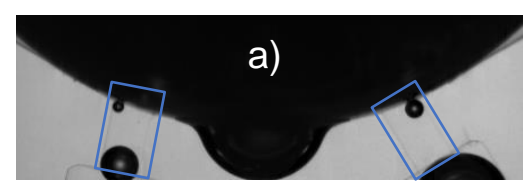

to

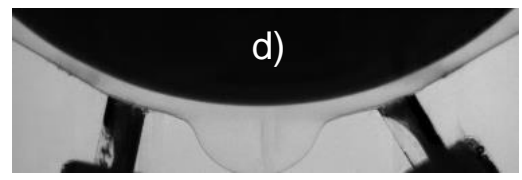

$\mathrm{t}_{0}+1 \mathrm{~ms}$

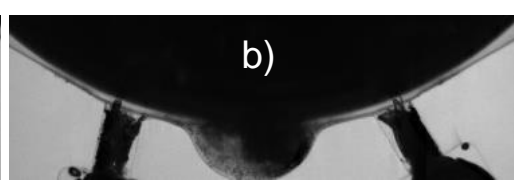

$\mathrm{t}_{0}+0.066 \mathrm{~ms}$

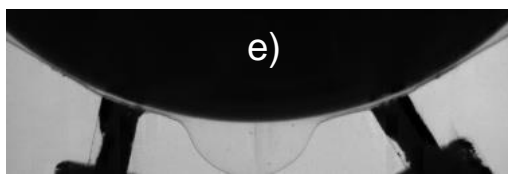

$\mathrm{t}_{0}+1.3 \mathrm{~ms}$

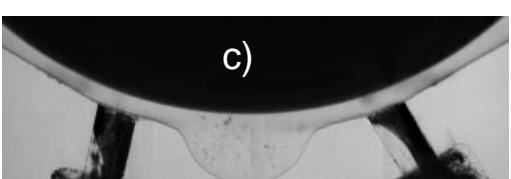

$\mathrm{t}_{0}+0.6 \mathrm{~ms}$

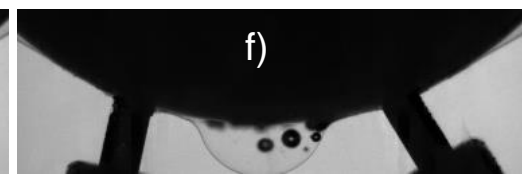

$\mathrm{t}_{0}+1.6 \mathrm{~ms}$

Figure 4. In-nozzle transient gas structures during an injection

\section{Injection into air and fuel}

In the following, we present the internal flow data focusing on the nozzle holes as highlighted by two rectangles in Figure 4a. As mentioned in the previous section, experiments were performed injecting fuel into both air and fuel environments at 1 bar backpressure in order to evaluate the impact of outside air entrained into the nozzle holes. Figure 5 shows exemplary single images before the injection and the probability of cavitation occurrence (calculated during the quasi steady phase of the injection according to Eq. 1) inside the holes in false color. As we see on the single images for the case of injection into liquid, no air bubbles are observed inside the nozzle holes before the injection phase.

$$
P=\frac{\sum_{i=1}^{N} p_{i}}{N}
$$

The average images for injection into liquid and gas exhibit a high similarity meaning that the air entrainment inside the holes is negligible at least during the steady-state injection phase evaluated here. Further internal flow measurements are carried out by injection into the liquid environment. 


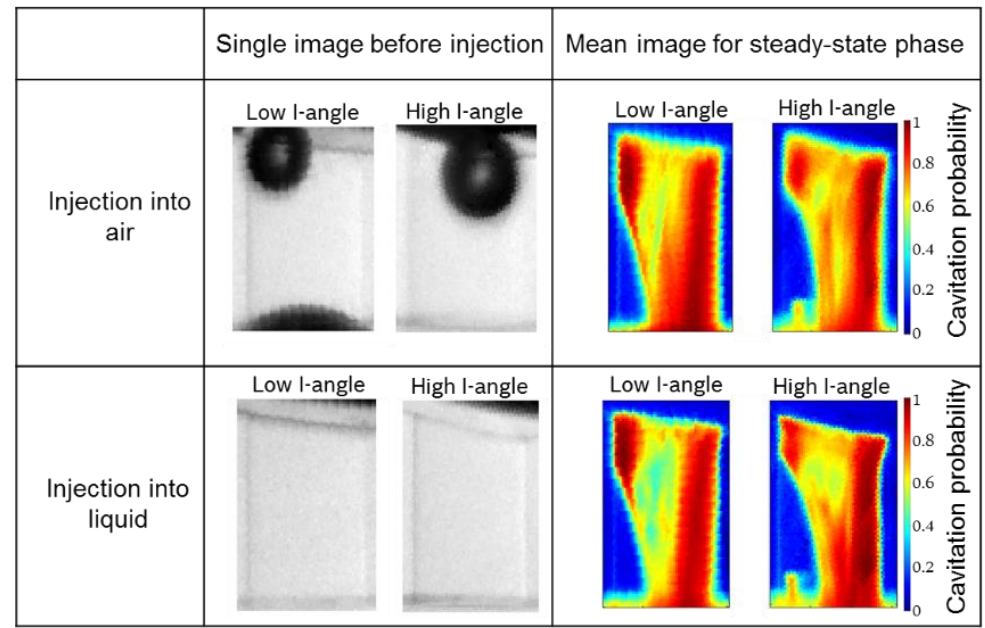

Figure 5. Comparison of injection into air and liquid fuel environment

\section{Effect of backpressure}

Figure 6 shows the development of cavitation for the nozzle with cylindrical holes against different backpressure levels. The injection pressure for these measurements was maintained at 100 bar. Only one nozzle hole (low Iangle) is presented in the pictures. The time gap between the sequential images is $8.3 \mu \mathrm{s}$. As can be observed, the gas structure inside the holes maintain their shape for the case of 1 bar backpressure, while rapid changes are seen for the higher backpressure conditions. The cavitation fraction seems to fluctuate and the rate of fluctuation tends to depend on the backpressure applied. The corresponding cavitation probability as well as the level and region of fluctuation in the images are presented on the right of Figure 6 . The cavitation probability is calculated according to Eq. 1; the fluctuation information is calculated based on the corresponding to steady-state injection phase (135 images) according to Eq. 2. Fluctuations are low where cavitation is almost always present and that the level of fluctuation is increased for higher backpressures. The region of cavitation fluctuation occupies a larger area for increasing backpressure. Possible reasons for this scenario could be that the cavitation film is thicker for lower backpressures. Due to the line-of-sight nature of shadowgraph imaging, the fluctuation can be observed only on the outer sides of the film. For higher backpressure, however, the cavitation film is thinner. Thus, the observable fluctuation area is larger. In addition, shear cavitation, which forms inside the counterbore, is inhibited for increased backpressure conditions to a high extent.

$$
R M S D=\sqrt{\frac{\sum_{i=1}^{N}\left(p_{i}-\bar{p}_{l}\right)^{2}}{N}}
$$

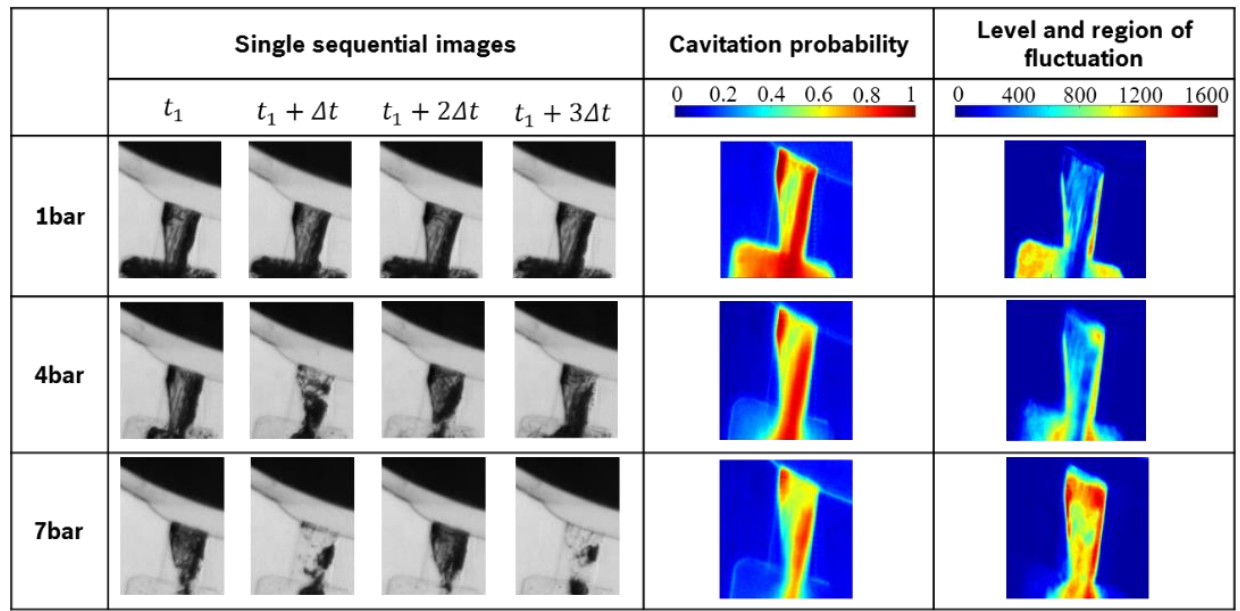

Figure 6. Cavitation under different backpressure levels during steady-state injection phase (cylindrical hole). Injection pressure -100 bar. 
Figure 7 shows the cavitation behavior in the nozzle with divergent holes under the same boundary conditions as Figure 6. From line-of-sight imaging, the holes appear to be relatively more affected by cavitation. From the sequential raw images, it can be observed that the fluctuations of the cavitation again increase with rising backpressure levels, although the cavitation seems to be more resistant against backpressure than for the case of cylindrical holes. Based on the mean images, it can be noticed that the average cavitation distribution and intensity is hardly changed for higher backpressures. There is also a relatively small change in the level and region of cavitation fluctuations. Evidentially, the divergent holes induce significantly higher levels of cavitation. The shear cavitation inside the counterbore, in turn, is also inhibited by increasing backpressures, but to a smaller degree than for cylindrical holes.

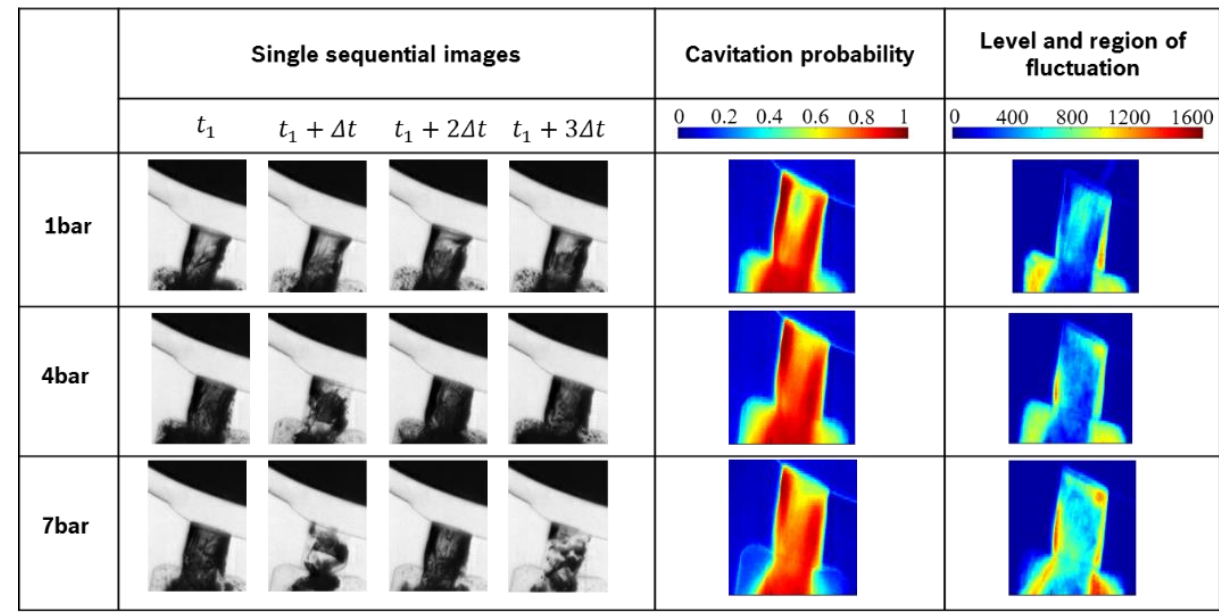

Figure 7. Cavitation under different backpressure levels during steady-state injection phase (divergent hole). Injection pressure - 100 bar.

In the following, the transient behavior of cavitation inside the nozzle holes under different backpressure conditions is analyzed and presented. Each nozzle image is cropped and rotated to obtain a vertical nozzle hole image for further analysis (see Figure 5). As a next step, the image is binarized by a fixed threshold in order to extract the cavitation contour and corresponding cavitation area in each nozzle hole. These 2D cavitation area changes are then tracked as shown in Figure 8. Afterwards, the resulting projected cavitation data is plotted over time. Figure 9 shows typical cavitating behavior of two cylindrical holes (low and high I-angle) over time after start of energization (SOE) for the case of back pressure of 7 bar. The data that corresponds to the quasi steady state of the injection is used for further evaluation. Within this quasi steady-state interval, the cavitation data is fitted with to a third order polynomial that is then subtracted from the data. This procedure is used to remove the long-term evolution of the cavitation pattern, while conserving all fast fluctuations for further Fast Fourier Transformation (FFT) analysis. However, before the FFT is applied, the data is multiplied by a Tukey windowing function in order to minimize spectral leakage. Figure 10 shows the result obtained by FFT transform for the measurement data depicted in Figure 9. Based on the FFT result, the dominant frequencies (depicted as 'peaks' in Figure 10) that correspond to the fluctuations of cavitation during the steady-state injection phase can be found. It should be kept in mind that the maximum frequency that can be recognized is determined by the Nyquist-Shannon sampling theorem and is $60 \mathrm{kHz}$ for the frame rate used for the measurements.

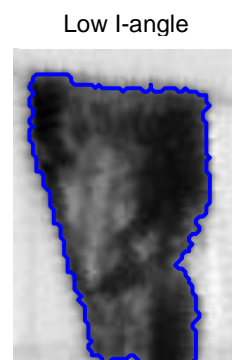

$\mathrm{t}_{1}$
Low I-angle

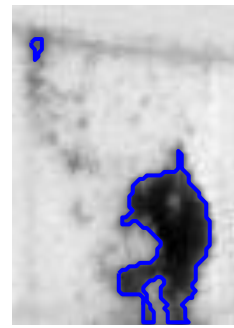

$\mathrm{t}_{1}+8.3 \mu \mathrm{s}$

Figure 8. Tracking of projected cavitation structures for two sequential images (cylindrical holes). Injection pressure - 100 bar, back pressure -7 bar 

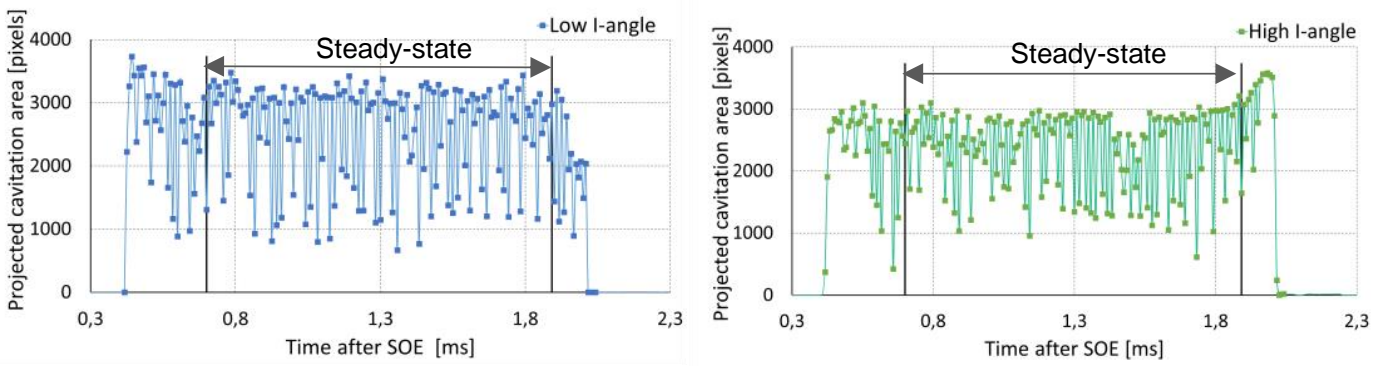

Figure 9. Projected cavitation area over time (cylindrical holes). Injection pressure -100 bar, backpressure -7 bar
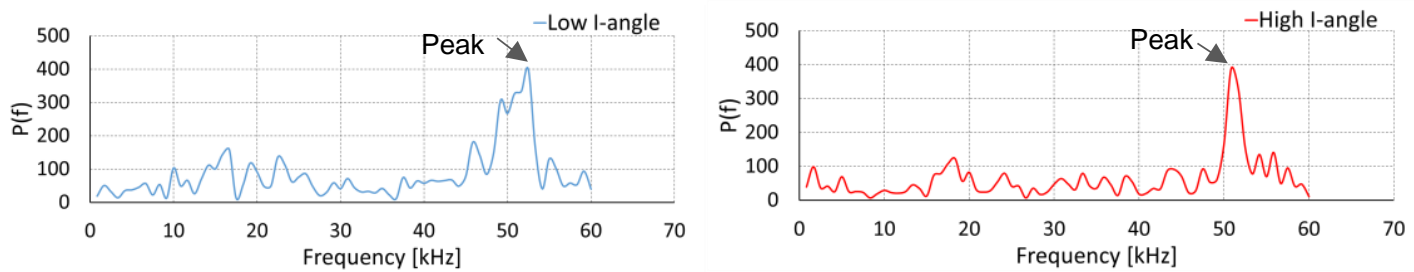

Figure 10. Spectral amplitude over frequency (cylindrical holes). Injection pressure -100 bar, backpressure -7 bar.

This procedure was performed for each backpressure condition and nozzle type. The results are combined and shown in Figure 11, where the dominant frequencies of cavitation fluctuations are depicted over the corresponding backpressure levels for each nozzle type and nozzle hole. For backpressures below 3 bar, no distinct dominant frequencies can be found; as a result, the lines on the graph start at 3 bar and continue up to 7 bar. Due to the fact that the experimental technique is based on the transmitted light principle, the cavitation analysis was performed for cavitation changes projected in $2 \mathrm{D}$ image plane over the injection process. It can be observed that the backpressure influences the frequency of cavitation fluctuations to a high extent for all nozzle holes measured. This graph confirms that divergent holes provide more robust cavitation structures (lower frequencies of cavitation fluctuations for the corresponding holes) than cylindrical ones. It can be observed that the holes under the low inclination angles are found to be subjected to cavitation fluctuations of higher frequencies than the holes under high inclination angles.

It is known that elevated backpressure inhibits the cavitation appearance [17]. Thus, the cavitation structures that appear, for instance, at the highly transient needle opening phase have more potential to collapse for higher back pressures. These collapses in turn generate pressure waves, which result in high pressure oscillations inside the nozzle and lead to cavitation instabilities. Further detailed theoretical interpretation of the observed cavitation fluctuations, however, would have to be performed as a continuation of this experimental study. Furthermore, the analysis and discussions of transient effects at the beginning and end of injection is to be carried out.

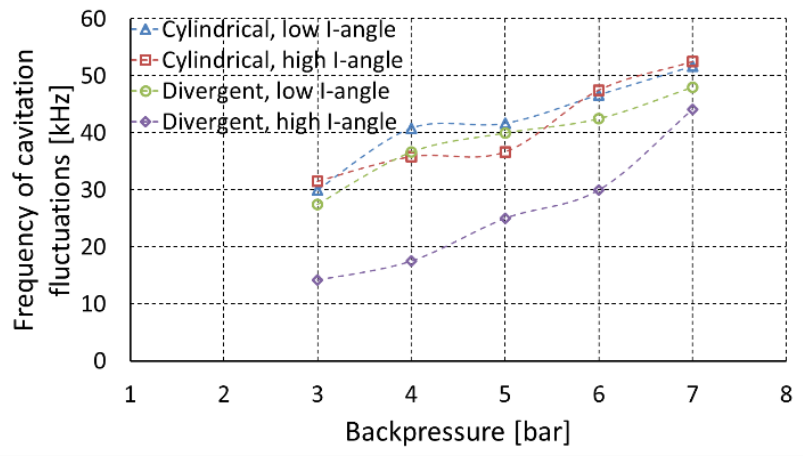

Figure 11. Dominant frequency of cavitation fluctuations vs. backpressure.

\section{Conclusions}

In this work, the internal cavitation behavior was visualized by means of optically accessible GDI nozzles and highspeed shadowgraphy. The detailed analysis of the transient cavitation characteristics was possible due to high temporal and spatial resolution of the images. The ambiguity between cavitation and ambient air drawn inside the nozzle holes was resolved by injecting into liquid fuel environment. The influence of drawn air was found to be 
negligible during the steady-state injection phase for the nozzle configuration used for this work. In addition, the cavitation behavior was investigated at elevated backpressures. It was found that the cavitation tends to fluctuate and the intensity of fluctuations is highly dependent on the backpressure being applied. It was shown that the divergent holes form cavitation structures that are more sustainable at elevated backpressure levels. The holes at low inclination angles, in turn, are subjected to higher fluctuations of cavitation.

\section{Acknowledgements}

The authors gratefully acknowledge the fruitful collaboration with Robert Bosch Corporate Research in Renningen. The invaluable advice of Dr. Kai Gartung, the expert support of Dr. Roman Grzeszik on the use of the high-speed shadowgraph imaging setup and the generous assistance of Andreas Herzig in the laboratory are greatly appreciated. This work could not have been completed without their support.

\section{Nomenclature}

$p_{i} \quad$ intensity of a, ' $i$ ' pixel [-]

$\bar{p}_{l} \quad$ average intensity of ' $i$ ' pixel over the steady-state phase of an injection [-]

$\mathrm{N}$ number of samples in a dataset [-]

$P \quad$ Probability [-]

RMSD Root-mean-square deviation [-]

\section{References}

[1] Payri, R., Molina, S., Salvador, F.J., Gimeno J., 2004, KSME International Journal, Vol. 18 No. 7, pp. 1222-1235. [2] Kufferath, A., Wiese, W., Samenfink, W., Dageförde, H., Knorsch, T., and Jochmann, P., March 10-11 2015, Fuel systems for IC engines.

[3] Knorsch, T., Rogler, P., Miller, M., and Wiese, W., Oct. 24-26 2016, SAE International Powertrains, Fuels \& Lubricants Meeting.

[4] Tamaki, N., Nishida K., and Hiroyasu, H., Jul. 6-8 1998, 14th International Conference on Liquid Atomization and Spray Systems.

[5] Sou, A., Hosokawa, S., Tomiyama, A., 2007, International Journal of Heat and Mass Transfer 50, pp. 35753582.

[6] Arcoumanis, C., Gavaises, M., Nouri, J. M., Abdul-Wahab, E. and Horrocks, R. W., 1998, SAE Technical Paper Series, 980811.

[7] Arcoumanis, C., Flora, H., Gavaises, M. and Badami, M., 2000, SAE Technical Paper Series, 200-01-1249.

[8] Aleiferis, P.G., Hardalupas, Y., Kolokotronis, D. and Taylor, A., Arioka, A., Saito, M., 2006, SAE Technical Paper Series, 2006-01-3374.

[9] Henry, M.E., Collicott, S.H., 2000, Atomization Sprays 10, pp. 545-563.

[10] Gilles-Birth, I., Rechs M. Spicher U., Bernhardt S., 2005, SAE Technical paper, 2005-01-3684

[11] Walther, J., Schaller, J. K., Wirth, R., and Tropea, C., 2000, Eighth International Conference on Liquid Atomization and Spray Systems, Pasadena, CA, USA.

[12] Miranda R., Chaves H., and Obermeier F., Sep. 9.-11. 2002, 18th European Conference on Liquid Atomization and Spray Systems.

[13] He, Z., Zhang, Z., Guo, G., Wang, Q., Leng, X., and Sun, S., 2016, International Communications in Heat and Mass Transfer 78 13-20.

[14] Gavaises, M., Andriotis, A., 2006, SAE Technical Paper Series 2006-01-1114.

[15] Balewski, B., Heine, B., Tropea, C., 2010, Atomization and Sprays, 20 (1) , pp. 57-50.

[16] Gilles-Birth, A.I., 2008, „Optische Untersuchung der Düseninnenströmung von Sitzlochinjektoren für die Benzindirekteinspritzung", Dissertation, Universität Karlsruhe.

[17] Tao, Q., Lei, Y., 2016, Fuel 173, pp. 79-89. 